Article

\title{
Incorporating Far-Infrared Data into Carbonate Mineral Analyses
}

\author{
Stephen Campbell and Kristin M. Poduska * \\ Department of Physics and Physical Oceanography, Memorial University of Newfoundland, \\ St. John's, NL A1B 3X7, Canada; scampbell@mun.ca \\ * Correspondence: kris@mun.ca
}

Received: 15 June 2020; Accepted: 10 July 2020 ; Published: 16 July 2020

\begin{abstract}
Polycrystalline carbonate minerals (including calcite, Mg-calcite, and aragonite) can show distinctive variations in their far-infrared (FIR) spectra. We describe how to identify mixed-phase samples by correlating FIR spectral changes with mid-infrared spectra, X-ray diffraction data, and simple peak overlap simulations. Furthermore, we show how to distinguish portlandite-containing $\left(\mathrm{Ca}(\mathrm{OH})_{2}\right)$ mixtures that are common in heated calcium carbonate samples. Ultimately, these results could be used for tracking how minerals are formed and how they change during environmental exposure or processing after extraction.
\end{abstract}

Keywords: calcite; aragonite; infrared spectroscopy; X-ray diffraction

\section{Introduction}

Calcium carbonate minerals $\left(\mathrm{CaCO}_{3}\right)$, including calcite and aragonite, are relevant for geoscience, archaeology, construction materials, and biomineralization [1-6]. Mixtures of these carbonate phases often exist together, and detecting the components that exist in these mixtures can be important for tracking how minerals are formed and how they change during environmental exposure or processing after extraction [5-8]. However, there are distinct challenges with these carbonates because there are multiple polymorphs of $\mathrm{CaCO}_{3}$ (calcite, aragonite, vaterite), and there is the tendency for amorphous or poorly crystalline phases to exist. This means that standard powder X-ray diffraction measures alone are not always sufficient to characterize carbonate mineral mixtures effectively.

A quick and easy way to assess small volumes of carbonate materials is infrared spectroscopy, which is one of the most widely used forms of vibrational spectroscopy. Not only are alloys and polymorphs often detectable from peak shifts, but amorphous forms of carbonate minerals also show distinctive infrared spectral variations in the mid-infrared (MIR) range [9]. This range of energies (400-4000 $\mathrm{cm}^{-1}$, which is equivalent to $12-120 \mathrm{THz}$ or $25-2.5$ microns) corresponds to vibrations within single carbonate moieties. Even in crystalline calcite, MIR spectra show distinctive peak-broadening trends that are correlated with crystallinity differences arising from greater microstrain fluctuation values and smaller crystalline domain sizes [8]. Earlier theoretical work demonstrated that analogous crystallinity differences do indeed trigger systematic peak broadening trends $[9,10]$.

Carbonates are not often investigated in the far-IR (FIR) energy range $\left(80-650 \mathrm{~cm}^{-1}\right.$, which is equivalent to $2.4-19.5 \mathrm{THz}$ or 125-15.4 microns), but FIR spectra can offer valuable insights about lower energy lattice vibrations that correspond to vibrations among — rather than within—different carbonate units. In principle, lattice modes should be subject to similar kinds of peak shifts, relative intensity differences, and broadening effects that are observed in the MIR energy range. However, there are gaps in the FIR knowledge base for two primary reasons. First, the spectrometer optical components required for this energy range must be different than the standard ionic alkali halide $(\mathrm{KBr})$ windows that absorb heavily in the FIR range. Second, theoretical calculations related to lattice mode energies 
are lacking because the quantum chemical calculations that work well to study MIR vibrations are not feasible for lattice modes with high dispersion [9].

In this work, we show how experimentally obtained FIR spectra can be a valuable part of carbonate composition analyses. To do this, we make new and explicit links between FIR and MIR spectra, and we support our findings with complementary X-ray diffraction data and simple, yet effective, peak overlap simulations.

\section{Materials and Methods}

\subsection{Calcite Crystal Structure and Vibrational Modes}

The crystal structure of calcite is often described with a hexagonal unit cell (space group $P \overline{3} 1 \mathrm{~m}$ ) that contains four formula units which are arranged in a way that produces alternating layers (in the $\mathrm{x}-\mathrm{y}$ plane) of calcium ions and carbonate units (which are rotated $60^{\circ}$ with respect to each other) [11]. The vibrational modes for calcite are typically derived from an analysis of its primitive rhombohedral unit cell which contains 10 atoms (two formula units). The energies and symmetries of calcite's 27 possible vibrational modes have been verified by many different theoretical and experimental studies [2,12-14]. There are four possible vibrations within a single carbonate moiety, but only three have a dipole moment that makes them IR-active. In the literature, these three modes have the following labels: $v_{2}$ designates out-of-plane bending, $v_{3}$ is asymmetric stretching, and $v_{4}$ is the in-plane bending or wagging. There are five additional IR-active lattice vibrational modes that are more challenging to describe because they involve relative translations of the calcium and carbonate ions. These lattice modes also have spectroscopic designations ( $v$ labels), but because some are close in energy, the spectral peaks associated with these lattice modes are usually reported as three bands: $300 \mathrm{~cm}^{-1}$ (comprised of $v_{6}$ and $v_{8}$, which we designate in this work as B1), $220 \mathrm{~cm}^{-1}$ (corresponding to $v_{9}$, which we call B2), and $100 \mathrm{~cm}^{-1}$ (comprised of $v_{7}$ and $v_{10}$, which we refer to as B3). Excellent animations of all IR-active local and lattice modes in calcite can be viewed online [10].

\subsection{Samples}

Table 1 lists nine samples that are the focus of this study. We used a purchased calcite $\left(\mathrm{CaCO}_{3}\right)$ powder (Alfa Aesar) as a reference. Other as-received samples were geogenic calcite (Woody Point, Newfoundland \& Labrador, Canada) and biogenic Mg-calcite (sea urchin, Newfoundland \& Labrador, Canada).

Heated samples $\mathrm{H} 1$ and $\mathrm{H} 2$ were formed by exposing limestone (calcite) to a wood fire outdoors [5]. H3, H4, and $\mathrm{H} 5$ were produced from calcium carbonate powders that were heated in a laboratory oven to temperatures of $800-900{ }^{\circ} \mathrm{C}$, then slaked with water. The chemistry of heat-treated and slaked calcite is well-documented in the production of lime binders, [6], with mixtures of calcite, aragonite, and portlandite $\left(\mathrm{Ca}(\mathrm{OH})_{2}\right)$ being characteristic products of this process. We note that, because the temperature-dependent sample fabrication process yields inherently mixed-phase samples, it is not feasible to separate and then quantify the overall aragonite and calcite content within these types of samples [5,6].

Aragonite (sample AR) was synthesized by a solution-based precipitation method involving $25 \mathrm{~mL} 60 \mathrm{mM} \mathrm{Na}_{2} \mathrm{CO}_{3}(\mathrm{pH}=10.9)$ added into $25 \mathrm{~mL} 60 \mathrm{mM} \mathrm{CaCl}_{2}(\mathrm{pH}=7.2)$ drop-by-drop over the span of three minutes while stirring at $400 \mathrm{rpm}$ and heating at $90^{\circ} \mathrm{C}$. After cooling to room temperature, the precipitates were separated from the suspension by centrifugation (4000 rpm for $10 \mathrm{~min}$ ), filtered, and dried at ambient temperature for $12 \mathrm{~h}$. 
Table 1. Summary of calcium carbonate samples used in this work.

\begin{tabular}{ll}
\hline Name & Description \\
\hline C1 & Purchased calcite \\
C2 & Geogenic calcite \\
M1 & Biogenic Mg-calcite \\
H1 & Field-heated calcite [5] \\
H2 & Field-heated calcite [5] \\
H3 & Lab-synthesized lime binder [15] \\
H4 & Lab-synthesized lime binder [15] \\
H5 & Lab-synthesized lime binder [15] \\
AR & Lab-synthesized aragonite \\
\hline
\end{tabular}

\subsection{Attenuated Total Reflectance IR Spectroscopy}

All data were collected using a Vertex 70v vacuum Fourier transform infrared (FTIR) spectrometer (Bruker, Billerica, MA, USA) with Platinum Diamond attenuated total reflectance (ATR) attachment and a globar (blackbody) light source. Measurements were made under vacuum with $2 \mathrm{~cm}^{-1}$ resolution. Due to absorbance characteristics of the spectrometer optics, data were collected two separate spectral ranges using either a $\mathrm{KBr}$ beam splitter $\left(4000-400 \mathrm{~cm}^{-1}\right)$, or a Mylar beam splitter $\left(650-80 \mathrm{~cm}^{-1}\right)$.

The data collection procedure involved repeated measurements, with regrinding by hand using a mortar and pestle following each. The rationale for using multiple grinding treatments for our ATR-FTIR measurements is based on previous experience that shows that grinding sharpens transmission FTIR spectra when preparing samples in diluted $\mathrm{KBr}$ pellets [5,16]. Although the scattering geometry for ATR samples is very different from samples diluted in KBr pellets, we observed similar ATR peak sharpening using successive grinding treatments. As large particle size are known to broaden ATR peaks, we use repeated grindings (typically 3-4 times) until the peak shapes show minimal changes, whether remounting and measuring the same powder, or by grinding and remeasuring a different portion of the same sample batch. Repeated measurements on the same powder showed variations of detected peak position $\pm 2 \mathrm{~cm}^{-1}$ (see Supplementary Materials File S1). This allows us to minimize sample-to-sample differences that could be caused by nonuniform particle size in an efficient and expedient way.

FTIR data in this work are displayed according to the common convention of ATR intensity vs. wavenumber, where the wavenumber axis is displayed with decreasing values from left to right.

\subsection{Powder X-ray Diffraction}

Powder X-Ray diffraction (PXRD) data were collected for a subset of samples using a Rigaku Ultima IV in $2 \theta / \theta$ system using a $\mathrm{Cu} \mathrm{K} \alpha$ source. The range $20^{\circ} \leq 2 \theta \leq 70^{\circ}$ was measured in continuous scanning mode, with a sampling width of 0.02 degrees and a scan speed of 1 degree $/ \mathrm{min}$. Prior to measurement, each sample ( $\sim 2 \mathrm{~g})$ was ground by hand using a mortar and pestle for several minutes. JADE software (MDI, Version 2010) [17] aided peak indexing and lattice constant refinements on z-corrected and background-subtracted spectra using 18 calcite peaks.

\section{Results}

Figure 1 shows combined MIR (2000-400 $\left.\mathrm{cm}^{-1}\right)$ and FIR $\left(650-80 \mathrm{~cm}^{-1}\right)$ data for the calcite-containing samples listed in Table 1 . All spectra show the three local vibrational modes of calcite ( $v_{3}$ near $1425 \mathrm{~cm}^{-1}, v_{2}$ at $875 \mathrm{~cm}^{-1}$, and $v_{4}$ at $712 \mathrm{~cm}^{-1}$ ) and the three lattice mode bands (band 1 (B1), near $300 \mathrm{~cm}^{-1}$ ( $v_{6}$ and $\left.v_{8}\right)$, band 2 (B2) centered near $220 \mathrm{~cm}^{-1}\left(v_{9}\right)$, and band 3 (B3) near $100 \mathrm{~cm}^{-1}$ $\left(v_{7}\right.$ and $\left.\left.v_{10}\right)\right)$. The energies of these vibrational modes have been verified by many different theoretical and experimental studies [2,12-14]. 


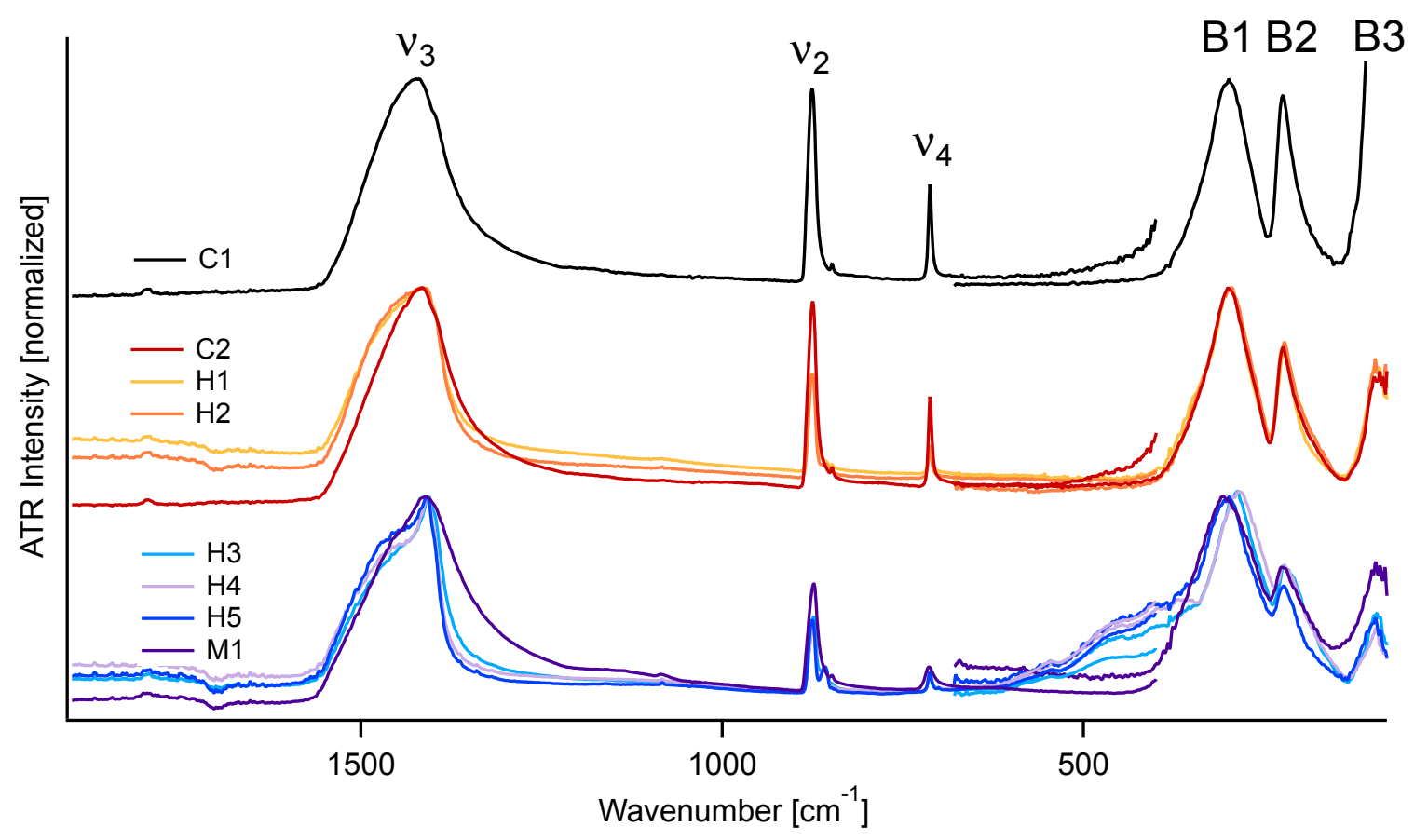

Figure 1. Representative mid-infrared (MIR) (2000-400 $\left.\mathrm{cm}^{-1}\right)$ and far-infrared (FIR) $\left(650-80 \mathrm{~cm}^{-1}\right)$ spectra for eight calcite-containing samples, shown on the same plot to demonstrate the extent and overlap of the spectral ranges. The intensity of each spectrum is normalized to either the $v_{3}$ peak (MIR configuration) or the B1 peak (FIR configuration). Spectra are grouped and offset for clarity.

The spectra in Figure 1 are grouped in a way that draws attention to similar types of features. Our calcite reference ( $\mathrm{C} 1$, black) is the top curve. Below that are two groupings. The middle group ( $\mathrm{C} 2, \mathrm{H} 1$, and $\mathrm{H} 2$, in shades of red) are most similar to the calcite reference, but with slightly broadened $v_{3}$ peaks and decreased B2 and B3 relative peak intensities. The bottom group (H3, H4, H5, M1, in shades of blue) has a sharp $v_{3}$ point, very distinctive broad shoulders from $550-350 \mathrm{~cm}^{-1}$, shifted B1 peaks, and very decreased B2 and B3 relative peak intensities.

In the following sections, we discuss the FIR data first, and then correlate our interpretations of the FIR spectral changes with MIR spectra, PXRD data, and peak overlap simulations.

\subsection{FIR-ATR}

Figure 2 gives an expanded view of the FIR $\left(600-80 \mathrm{~cm}^{-1}\right)$ spectra, each of which is normalized to its own B1 peak. The reference calcite (C1) and the middle grouping (H1, H2, C2) have nearly identical spectral band positions and widths for B1 and B2. The main difference with the reference calcite is that it has a higher relative B2 band intensity. We do not attempt to ascribe relative intensity comparisons for band B3 because that spectral feature extends below our low-wavenumber detection limit.

In contrast, the bottom set of spectra (H5, H4, H3, M1) show much more variation in their spectral features. In all cases, B1 broadens and shows slight position shifts relative to the reference calcite. Although the position of band B2 remains consistent, that peak is reduced in its relative intensity. Furthermore, samples H3, H4, and H5 have a broad shoulder on the high-wavenumber side of the B1 peak that extends well into the MIR region. 


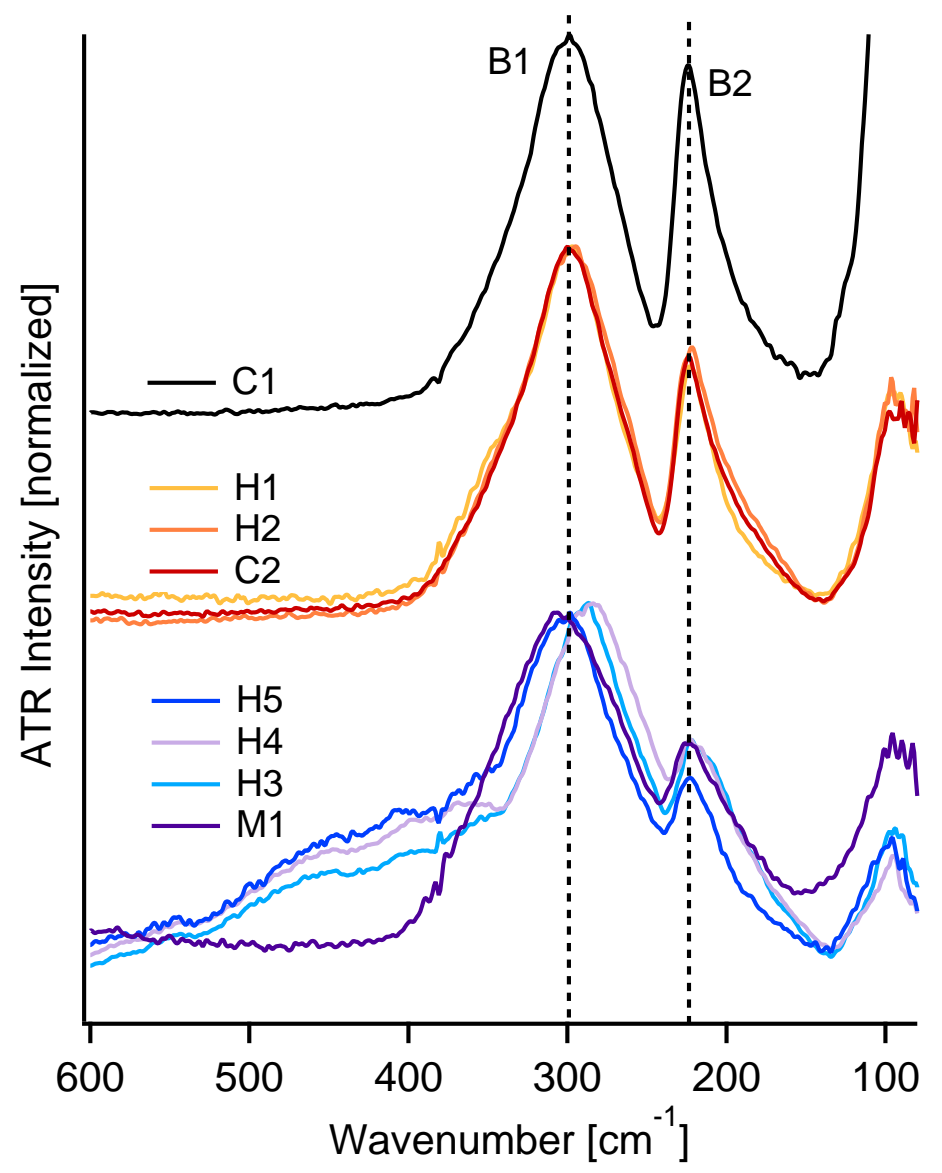

Figure 2. Representative FIR spectra $\left(600-80 \mathrm{~cm}^{-1}\right)$ for the same samples shown in Figure 1. Here, the intensity of each spectrum is normalized to the B1 peak, and the dashed lines denote the positions of the B1 and B2 peak maxima in the spectrum for the reference calcite. Spectra are grouped and offset for clarity.

\subsection{MIR-ATR}

To explain the FIR spectral differences, it is helpful to link our observations described above with MIR data for the same samples. Figure 3 shows enlarged views of four key MIR regions.

The hydroxide region (Figure 3a) shows minor water vapor vibrations for all samples in the $4000-3400 \mathrm{~cm}^{-1}$ range. This is a common spectral feature for calcium carbonate powders such as ours that were not desiccated prior to measurement. For samples $\mathrm{H} 3, \mathrm{H} 4$, and $\mathrm{H} 5$, there are additional peaks near $3640 \mathrm{~cm}^{-1}$ that are characteristic of portlandite $\left(\mathrm{Ca}(\mathrm{OH})_{2}\right)$, which is a well-known intermediate phase that exists in these kinds of lime binder specimens [6].

The same samples that show evidence of $\mathrm{Ca}(\mathrm{OH})_{2}$ in Figure 3a also show distinctive $v_{3}$ peak shapes relative to the other samples. In Figure 3b, H3, H4, H5 (in the bottom grouping) show a sharp peak on the low-wavenumber side of the $v_{3}$ band. This band shape has been reported previously in FTIR reflectance spectra of lime plasters, ref. [18] but it is not as noticeable in transmission FTIR spectra [16]. In contrast, our reference calcite (C1) shows a broad featureless peak centered near $1425 \mathrm{~cm}^{-1}$. Spectra in the middle grouping (H1, H2, C2) show asymmetric broadening on the high-wavenumber side of the $v_{3}$ band; on the other hand, M1 shows extreme broadening on the low-wavenumber side of the $v_{3}$ peak.

The $v_{2}$ and $v_{4}$ peaks (Figure $3 c, d$, respectively) give further insights into the origins of the spectral differences. Our reference calcite (C1) shows characteristic peaks centered at $875 \mathrm{~cm}^{-1}$ and $712 \mathrm{~cm}^{-1}$, along with a smaller peak corresponding to the isotopic ${ }^{13} \mathrm{C} v_{2}$ vibrational mode at $850 \mathrm{~cm}^{-1}$ [19]. Heated samples $\mathrm{H} 1$ and $\mathrm{H} 2$ have the same peak positions as the calcite reference with an additional 
weak peak at $858 \mathrm{~cm}^{-1}$ due to aragonite. The relative intensities of the calcite peaks are reduced compared to the reference. Heated samples $\mathrm{H} 3, \mathrm{H} 4$, and $\mathrm{H} 5$ show a further weak peak at $700 \mathrm{~cm}^{-1}$, also due to aragonite, as well as the $858 \mathrm{~cm}^{-1}$ peak found in the other heated samples. Thus, the heated samples with the most distinctive MIR and FIR spectra are a calcite-rich mixture that contains both aragonite and portlandite as secondary phases.
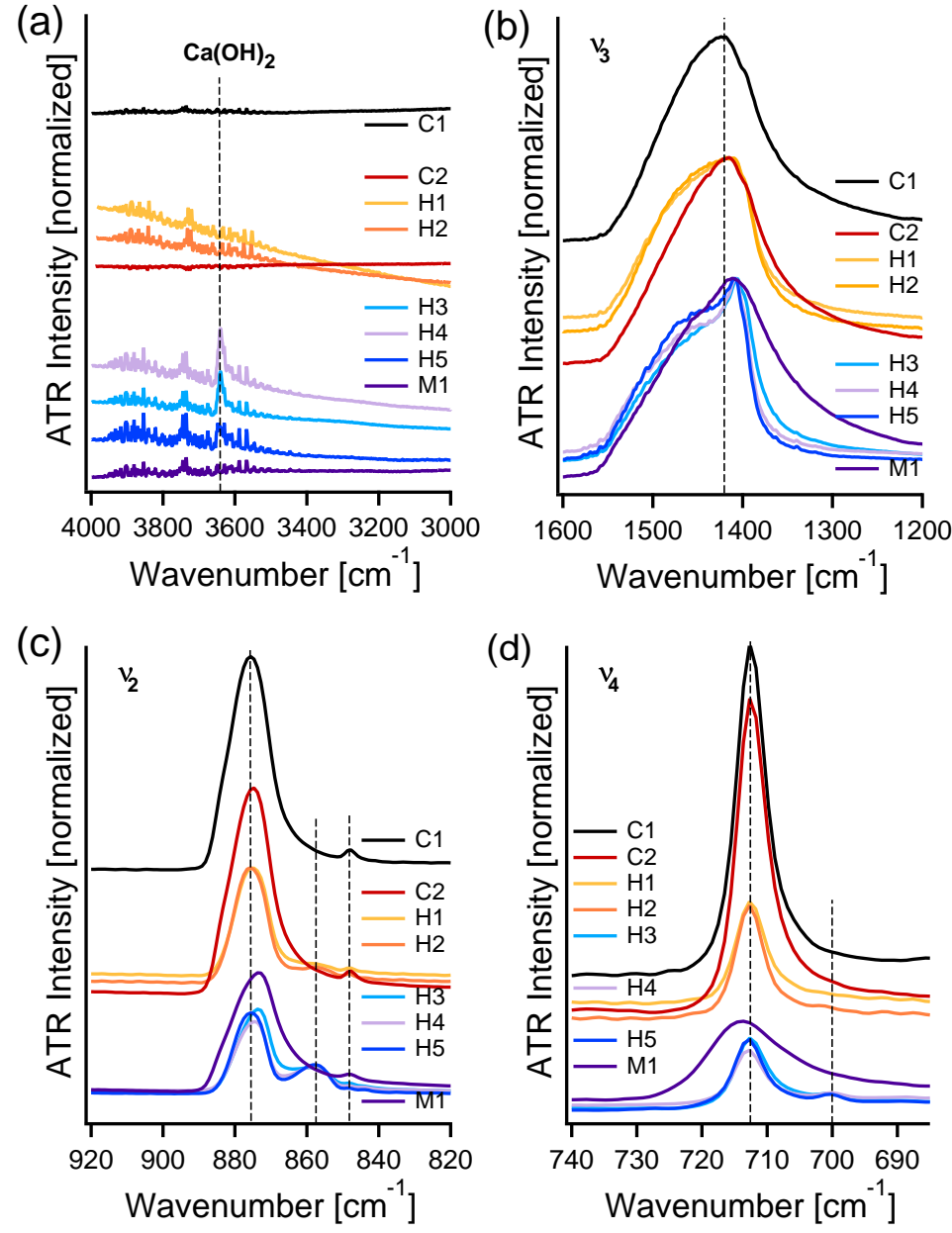

Figure 3. Representative MIR spectra, highlighting regions related to (a) $\mathrm{OH},(\mathbf{b}) v_{3}$, (c) $v_{2}$, and (d) $v_{4}$ peaks. Each spectrum is normalized to the intensity of the $v_{3}$ peak, and spectra are offset and grouped for clarity. In (a), the dominant portlandite $\left(\mathrm{Ca}(\mathrm{OH})_{2}\right)$ peak position is shown with a dashed line; other peaks are related to water vapor. In (b), the dashed line corresponds to the calcite $v_{3}$ maximum. In (c), the dashed lines correspond to calcite $v_{2}\left(875 \mathrm{~cm}^{-1}\right)$, aragonite $v_{2}\left(858 \mathrm{~cm}^{-1}\right)$, and calcite ${ }^{13} \mathrm{C} v_{2}$ $\left(850 \mathrm{~cm}^{-1}\right)$. In $(\mathrm{d})$, the dashed lines correspond to calcite $v_{4}\left(712 \mathrm{~cm}^{-1}\right)$ and aragonite $v_{4}\left(700 \mathrm{~cm}^{-1}\right)$.

The biogenic M1 sample shows a broadened $v_{2}$ peak as well as a $v_{4}$ peak that is broad and shifted to slightly higher wave numbers. This $v_{4}$ shift is characteristic of $\mathrm{Mg}$-containing calcite, wherein the lighter mass $\mathrm{Mg}$ leads to a higher wavenumber $v_{4}$ peak position [5].

\subsection{PXRD}

PXRD data were collected for the subset of samples that appeared to be closest to pure calcite $(\mathrm{C} 1, \mathrm{H} 1, \mathrm{H} 2)$ to look for evidence of secondary phases or structural differences within the calcite portion of the sample. As an example, Figure 4 shows two cases where aragonite appeared as a minor secondary phase. Close inspection of the MIR data (Figure 3c,d) confirms a small aragonite peak at $858 \mathrm{~cm}^{-1}$. The presence of aragonite is not surprising given the previous high-temperature exposure of these samples [5]. 
Focusing on the dominant calcite phase, we extracted lattice constants for the conventional hexagonal unit cell (Table 2) to determine that $\mathrm{H} 1, \mathrm{H} 2$, and $\mathrm{C} 2$ have comparable $a$ lattice constants, but that the heated samples have slightly contracted $c$ lattice constants. We attempted Williamson-Hall (WH) analyses to extract crystallite size and microstrain fluctuation values, but the results proved to be inconclusive, likely due to instrumental broadening dominating the widths of our diffraction peaks (see Supplementary Materials File S1).

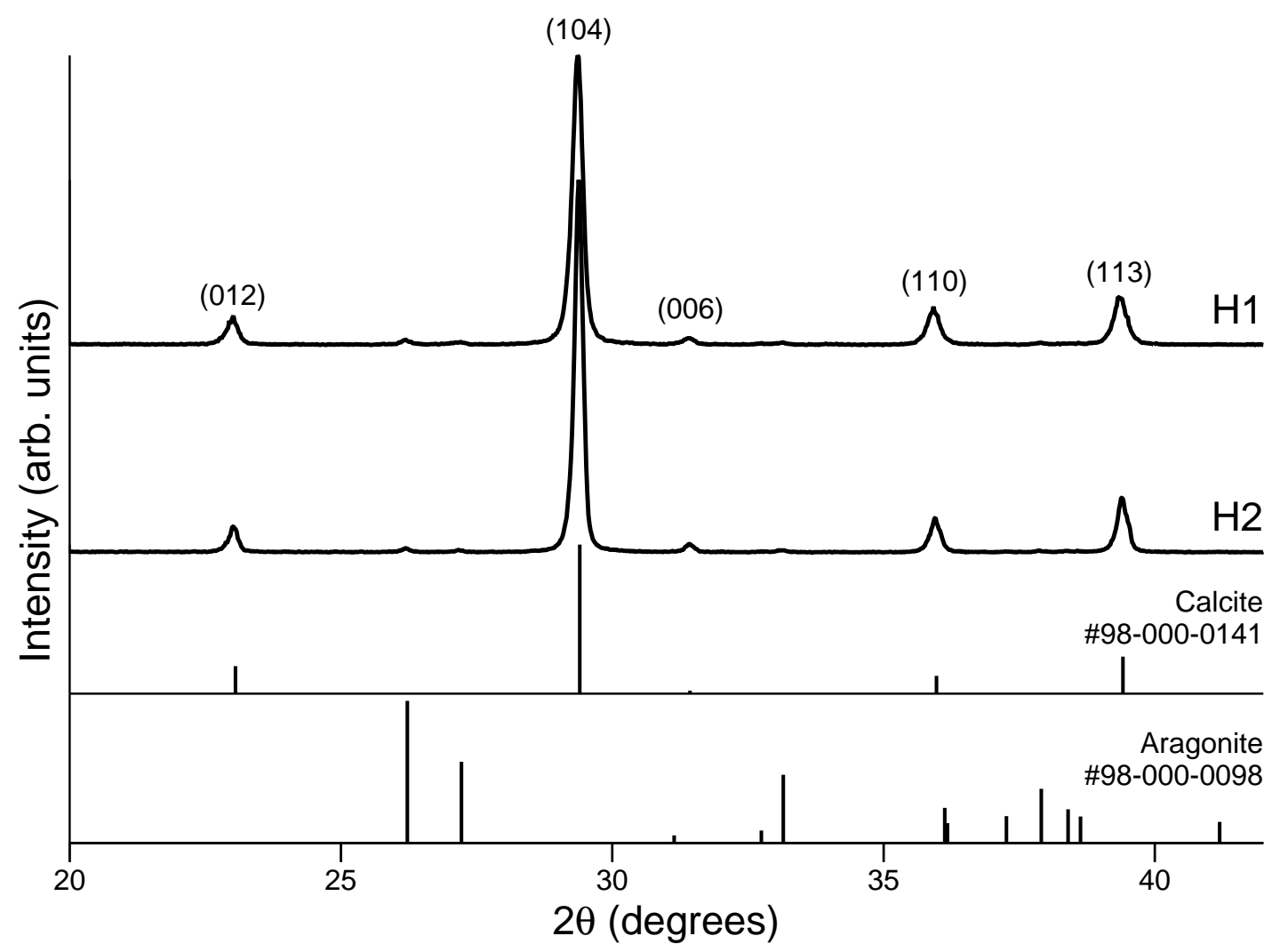

Figure 4. Powder X-Ray diffraction (PXRD) data for $\mathrm{H} 1$ and $\mathrm{H} 2$ samples, with calcite peak $h k l$ indices labeled. JCPDS references for calcite peaks and aragonite are included as bars below the diffraction data [11].

Table 2. Refined lattice constants for the conventional hexagonal calcite unit cell. Uncertainty estimates, based on whole pattern fitting with at least 18 calcite peaks, are shown in parentheses. The literature value corresponds to JCPDS powder diffraction file 98-000-0141 [11].

\begin{tabular}{lcc}
\hline Name & a $(\AA)$ & c $(\AA)$ \\
\hline Literature [11] & 4.989 & 17.061 \\
C1 & $4.9895(4)$ & $17.061(1)$ \\
H1 & $4.988(1)$ & $17.049(2)$ \\
H2 & $4.990(1)$ & $17.048(2)$ \\
\hline
\end{tabular}

\section{Discussion}

Previous work examining calcium carbonate vibrations in the FIR/THz energy range $\left(650 \mathrm{~cm}^{-1}-80 \mathrm{~cm}^{-1}\right)$ is sparse. Nevertheless, we are aware of two detailed studies that focused on identifying mode energies for a wide range of carbonate minerals, [2]; and identifying Mg-containing carbonate alloy phases [20].

Brunsentsova et al. report FIR spectra for calcite, dolomite $\left(\mathrm{Ca}_{0.5} \mathrm{Mg}_{0.5} \mathrm{CO}_{3}\right)$, and magnesite $\left(\mathrm{MgCO}_{3}\right)$, and their data show that the B1 band shifts to higher wavenumbers with higher amounts 
of Mg incorporation [2]. In a separate study, Sakai et al. used Terahertz Time-Domain Spectroscopy (THz-TDS) to collect calcium carbonate lattice vibrational data for the $1-6 \mathrm{THz}\left(30-200 \mathrm{~cm}^{-1}\right)$ range, which includes the $\mathrm{B} 3$ band $\left(100 \mathrm{~cm}^{-1}\right)$ [20]. They reported that changes in B3 intensity could be used to differentiate among high- and low-Mg calcites, and that the intensity of that band was also influenced by relative amounts of calcite and aragonite in mixed-phase samples.

In the sections below, we put our observed peak position changes and relative intensity changes in the context of this previous work to make explicit links between FIR, MIR, and PXRD studies of calcium carbonate and related minerals. In doing so, we pay special attention to identifying phase mixtures in carbonate minerals that have been exposed to high temperatures, since this has not been reported by others.

\subsection{FIR Peak Positions}

In our FIR spectra (Figure 2), it is the region near the B1 band that shows the most variation among different samples.

The most obvious difference is the broad shoulder that extends from $350-550 \mathrm{~cm}^{-1}$ in a subset of the heated samples $(\mathrm{H} 3, \mathrm{H} 4, \mathrm{H} 5)$. We attribute this feature to portlandite $\left(\mathrm{Ca}(\mathrm{OH})_{2}\right)$, since it correlates perfectly with MIR spectra peaks that are consistent with $\mathrm{Ca}(\mathrm{OH})_{2}$ (Figure 3a). As noted earlier, $\mathrm{Ca}(\mathrm{OH})_{2}$ is a well-known intermediate phase that appears in lime binders [5,6], so it is not surprising to find this phase in our heated samples. We note that the $\mathrm{Ca}(\mathrm{OH})_{2}$-related shoulder in FIR spectra is much easier to identify than the weak peaks that appear for this hydroxide phase in the mid-IR region (near $3640 \mathrm{~cm}^{-1}$ ) in Figure 3a). In this way, it is much easier to detect portlandite in FIR spectra than in MIR spectra. However, the fact that this feature is a broad shoulder rather than a peak means that we do not assign a specific wavenumber to this feature.

Among the heated samples (H1-5), another distinct trend in the FIR spectra is that the B1 band position varies, while the B2 band does not. Comparing with data in the MIR range, these heated samples do not show any shifts in their calcite-related peaks (Figure 3c,d). However, all heated samples show evidence of aragonite as a secondary phase, based on MIR features (H1-5 in Figure 3c,d) or PXRD data (H1, H2 in Figure 4).

We attribute this apparent shift of the B1 peak to the aragonite-calcite phase mixtures in these heated samples. Previous work by others shows that calcite and aragonite each have broad B1 bands that overlap in energy [2]. To demonstrate the implications of this, Figure 5a shows how a change in relative intensity between two broad, overlapping peaks can lead to a continuous shift in the energy position of the maximum of the combined peaks. Applying this idea to our samples, a more aragonite-rich mixture could cause the B1 band maximum to shift to lower wavenumber values.

Although the simulated peak overlaps in Figure 5a are a simple mathematical exercise, it shows excellent qualitative agreement with the B1 peak changes that we observe in our mixed calcite-aragonite samples. Samples $\mathrm{H} 1$ and $\mathrm{H} 2$ have lesser amounts of aragonite (much lower aragonite MIR peaks intensities, but aragonite present in PXRD data) and show a B1 shift that is so small that it is near our minimum detectable shift (see Supporting Material). More apparent B1 peak shifts occur for $\mathrm{H} 3$ and H4, both of which have higher relative aragonite peak intensities (Figure 3c,d). We note that H5 has a higher amount of aragonite, yet does not have a discernible B1 band shift. In this case, we suspect that the relatively high amount of $\mathrm{Ca}(\mathrm{OH})_{2}$ may be a contributor to the $\mathrm{B} 1$ band, which could disrupt the simple trend that we suggest for aragonite:calcite mixtures. It is possible that the band position change is dominated by the portlandite contribution, while the overall band shape change is related to the aragonite. Thus, less portlandite could mean a peak shape change with minimal (or no) B1 shift.

Our comparisons in Figure 5 highlight a simple-but important-distinction that makes interpreting FIR spectral trends very different than what has been reported for carbonates in the MIR. In this work, we show that apparent peak shifts may occur due to phase mixing (contamination) resulting in changes to the relative intensities of overlapping broad peaks. This is important because a peak shift in MIR and FIR spectra is generally attributed to an alloy (composition) change [2,5]. 
(a)

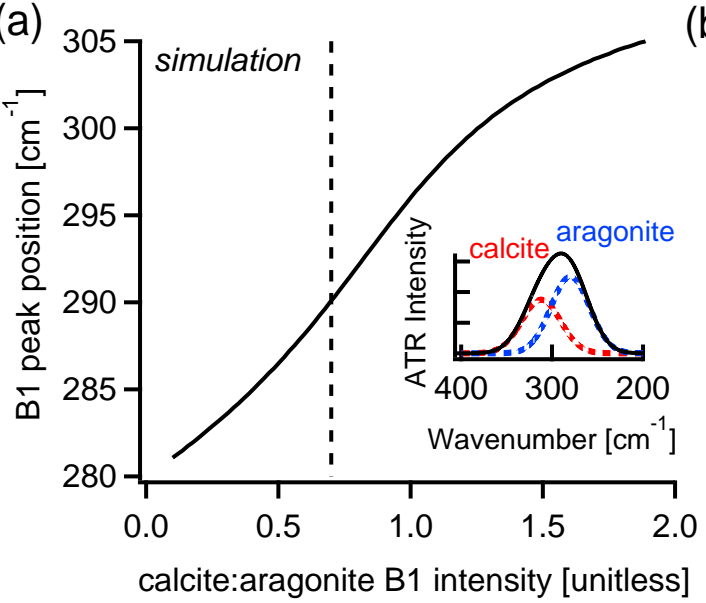

(b)

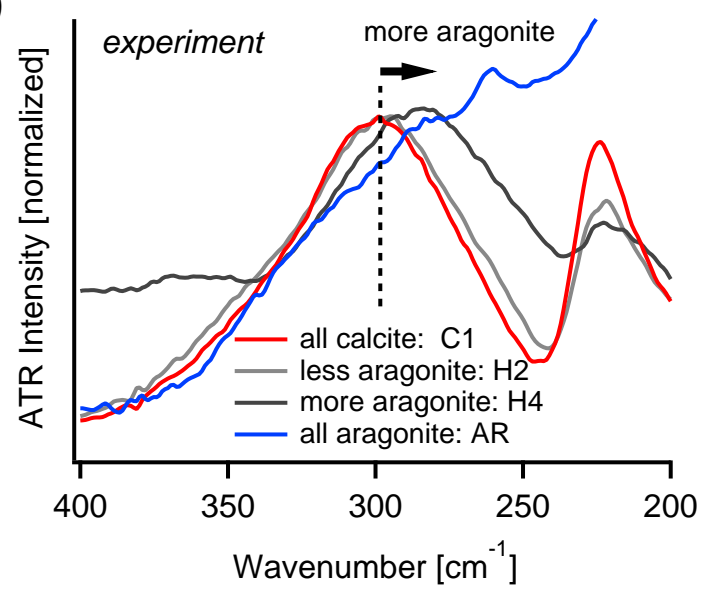

Figure 5. (a) A simulation based on a linear combination of two broad, overlapping Gaussian peaks highlights the effect of changing relative peak intensities on the resulting position of the peak maximum. The inset shows two Gaussian peaks (dashed) centered at $312 \mathrm{~cm}^{-1}$ and $280 \mathrm{~cm}^{-1}$, and the resultant linear combination (black) with a position of $290 \mathrm{~cm}^{-1}$. Correspondingly, a dashed line vertical line shows that a calcite:aragonite ratio near 0.7 leads to a B1 position of $290 \mathrm{~cm}^{-1}$. In this simulation, changing the relative calcite:aragonite B1 intensity causes a monotonic change in the resulting B1 peak position. (b) Experimental data show a shift of band B1 (near $300 \mathrm{~cm}^{-1}$ ) to lower wavenumbers for calcite-aragonite mixtures.

Finally, we had one unique sample in our FIR data: the spectrum for biogenic M1 shows a slight B1 band shift to higher wavenumbers. Earlier FIR studies of Mg-containing calcite-type minerals (dolomite and magnesite) showed that the B1 band moves to higher wavenumbers with higher levels of $\mathrm{Mg}$ incorporation [2]. Recent THz-TDS studies of high- and low-Mg calcite alloys focus on lower energy lattice modes (B3 and others) and do not cover this higher-energy B1 mode [20]. Fortunately, there is extensive MIR literature for Mg-containing calcites, and the blue-shift and broadening of the MIR $v_{4}$ mode that we observe (Figure $3 \mathrm{~d}$ ) is entirely consistent with low $(<10 \%) \mathrm{Mg}$ incorporation into calcite, as is common for marine biogenic specimens like our sea urchin shell [5]. Thus, we attribute the $\mathrm{B} 1$ band shift for our biogenic M1 sample to Mg incorporation.

\subsection{FIR Relative Peak Intensities}

We note that there are as of yet no comparable quantum chemical calculations that allow us to track changes in FIR peak positions or relative intensities; this is distinctly different from transmission MIR experiments, which have the benefit of quantum chemical calculations to back them $[9,10,16,19]$. This knowledge gap in the FIR is related in part to the computational expense of the large supercells required to accommodate lattice vibrational modes, and also in part to the inherent energy dispersion of lattice modes [9].

There is also a very important distinction between the work we present here (based on ATR-FTIR) and these previous transmission MIR data: ATR peaks have different line profiles and relative intensity profiles compared with spectra collected from diluted powders that are well-dispersed in a KBr pellet for transmission FTIR measurements [18]. These spectral differences between ATR and transmission IR data may seem small, but our experience shows us that they are important. The first challenge is that the peaks in ATR spectra are visibly asymmetric, which makes it unwise to use peak deconvolution with Gaussian, Lorentzian, or pseudo-Voigt functions. In contrast, transmission IR peaks are often well-enough approximated by Gaussian functions to allow for peak deconvolution. The second challenge is that our bands B1 and B3 are known to be comprised of more than one vibrational mode, but their energies are so close-relative to the width of the peaks-that it is not feasible to resolve individual peaks. 
We note that previous MIR studies of calcites have utilized grinding curves to quantify differences in peak intensity ratios $[5,7,16]$. As tempting as it is to explore, grinding curves are not rigorously applicable to our ATR-FTIR data. Nevertheless, we find that our MIR ATR data have qualitatively similar relative peak intensities to the transmission MIR data: $v_{3}$ has higher intensity than $v_{2}$ which has higher intensity than $v_{4}$. Thus, we make only qualitative peak intensity comparisons in our FIR-ATR spectra by analogy with transmission MIR data that has been verified by quantum chemical calculations.

The most robust relative peak intensity differences occur between the B1 and B2 bands $\left(300 \mathrm{~cm}^{-1}\right.$ and $220 \mathrm{~cm}^{-1}$, respectively, in Figure 2). $\mathrm{H} 1$ and $\mathrm{H} 2$ show a larger relative B2 intensity compared to the other heated samples (H3, H4, H5); all heated samples have a smaller relative B2 intensity compared to the calcite reference $\mathrm{C} 1$. We note that these groupings of FIR relative peak intensity trends mirror the relative peak intensity trends that we see in MIR data $\left(v_{2}\right.$ and $\left.v_{4}\right)$ for the same samples. Thus, although we can see similar changes in the FIR B2:B1 that qualitatively mirror the MIR $v_{4}: v_{2}$, these data do not allow us to determine if the relative intensity differences are related to compositional differences or crystallinity differences. The limitations we encounter in evaluating examining crystallinity-related lattice mode changes in our mixed phase samples could potentially be addressed in a future study by using carefully controlled pure-phase samples to assess spectral changes due to crystallinity differences more definitively.

\section{Conclusions}

Our explicit links between FIR and MIR data are the first of its kind, and help move us toward our ultimate goal of using spectroscopic analysis strategies for tracking structural differences in materials that are due to processing, aging, or use-based changes. We demonstrate that calcite-aragonite-portlandite mixtures can show apparent peak shifts in FTIR-ATR spectra (650-80 $\left.\mathrm{cm}^{-1}\right)$. We show that relative peak intensity changes between overlapping peaks-without peak energy shifts in the constituent peaks-are sufficient to explain peak position changes. These results are particularly useful in heat-treated calcium carbonate samples wherein phase mixtures are common.

Supplementary Materials: The following are available online at http:/ / www.mdpi.com/2075-163X/10/7/628/ s1, File S1: Supporting materials for "Incorporating Far- Infrared Data into Carbonate Mineral Analyses".

Author Contributions: Conceptualization, resources, supervision, project administration, and funding acquisition: K.M.P.; methodology, formal analysis, writing-review and editing: S.C. and K.M.P.; laboratory experiments, data curation, writing-original draft preparation: S.C. Both authors have read and agreed to the published version of the manuscript.

Funding: This research and the article processing charges are funded by the Natural Sciences and Engineering Research Council (Canada) Discovery Grant number 2018-04888.

Acknowledgments: Thanks to L. Regev (Weizmann Institute of Science), M. Toffolo (Université Bordeaux Montaigne), and B. Gao (Memorial University) for carbonate samples, thanks to J. Aimee (Memorial University) for coding the initial stages of the peak overlap simulation. PXRD data were collected through the CREAIT network (Memorial University) with the help of W. Aylward.

Conflicts of Interest: The authors declare no conflict of interest. The funders had no role in the design of the study; in the collection, analyses, or interpretation of data; in the writing of the manuscript, or in the decision to publish the results.

\section{References}

1. Hussin, M.; Bhutta, M.; Azreen, M.; Ramadhansyah, P.; Mirza, J. Performance of blended ash geopolymer concrete at elevated temperatures. Mater. Struct. 2015, 48, 709-720. [CrossRef]

2. Brusentsova, T.N.; Peale, R.E.; Maukonen, D.; Harlow, G.E.; Boesenberg, J.S.; Ebel, D. Far infrared spectroscopy of carbonate minerals. Am. Mineral. 2010, 95, 1515-1522. [CrossRef] 
3. Zhu, Y.; Ma, N.; Jin, W.; Wu, S.; Sun, C. Genomic and transcriptomic insights into calcium carbonate biomineralization by marine actinobacterium Brevibacterium linens BS258. Front. Microbiol. 2017, 8, 602. [CrossRef] [PubMed]

4. Pan, J.; Zhao, H.; Tucker, M.E.; Zhou, J.; Jiang, M.; Wang, Y.; Zhao, Y.; Sun, B.; Han, Z.; Yan, H. Biomineralization of Monohydrocalcite Induced by the Halophile Halomonas Smyrnensis WMS-3. Minerals 2019, 9, 632. [CrossRef]

5. Regev, L.; Poduska, K.M.; Addadi, L.; Weiner, S.; Boaretto, E. Distinguishing between calcites formed by different mechanisms using infrared spectrometry: Archaeological applications. J. Archaeolog. Sci. 2010, 37, 3022-3029. [CrossRef]

6. Toffolo, M.B.; Boaretto, E. Nucleation of aragonite upon carbonation of calcium oxide and calcium hydroxide at ambient temperatures and pressures: A new indicator of fire-related human activities. J. Archaeolog. Sci. 2014, 49, 237-248. [CrossRef]

7. Xu, B.; Poduska, K.M. Linking crystal structure with temperature-sensitive vibrational modes in calcium carbonate minerals. Phys. Chem. Chem. Phys. 2014, 16, 17634-17639. [CrossRef] [PubMed]

8. Xu, B.; Toffolo, M.B.; Regev, L.; Boaretto, E.; Poduska, K.M. Structural differences in archaeologically relevant calcite. Anal. Methods 2015, 7, 9304-9309. [CrossRef]

9. Gueta, R.; Natan, A.; Addadi, L.; Weiner, S.; Refson, K.; Kronik, L. Local atomic order and infrared spectra of biogenic calcite. Angew. Chem. Int. Ed. 2007, 46, 291-294. [CrossRef] [PubMed]

10. Valenzano, L.; Noel, Y.; Orlando, R.; Zicovich-Wilson, C.; Ferrero, M.; Dovesi, R. Ab initio vibrational spectra and dielectric properties of carbonates: magnesite, calcite and dolomite. Theor. Chem. Acc. 2007, 117, 991-1000. [CrossRef]

11. Gates-Rector, S.D.; Blanton, T.N. The Powder Diffraction File: A Quality Materials Characterization Database. Powder Diffr. 2019, 34, 352-360. [CrossRef]

12. Lou, D.; Sun, F.; Li, L. Study on vibrational modes by group theory and infrared spectra by DFT for calcite crystal. Chin. Opt. Lett. 2007, 5, 370-372.

13. Jiang, C.L.; Zeng, W.; Liu, F.S.; Tang, B.; Liu, Q.J. First-principles analysis of vibrational modes of calcite, magnesite and dolomite. J. Phys. Chem. Solids 2019, 131, 1-9. [CrossRef]

14. White, W.B. The Carbonate Minerals. In The Infrared Spectra of Minerals; Mineralogical Society of Great Britain and Ireland: Twickenham, UK, 1974. [CrossRef]

15. Xu, B.; Toffolo, M.B.; Boaretto, E.; Poduska, K.M. Assessing local and long-range structural disorder in aggregate-free lime binders. Ind. Eng. Chem. Res. 2016, 55, 8334-8340. [CrossRef]

16. Poduska, K.M.; Regev, L.; Boaretto, E.; Addadi, L.; Weiner, S.; Kronik, L.; Curtarolo, S. Decoupling local disorder and optical effects in infrared spectra: differentiating between calcites with different origins. Adv. Mater. 2011, 23, 550-554. [CrossRef] [PubMed]

17. MDI. JADE; Version 2010; Computer Software; Materials Data: Livermore, CA, USA, 2010.

18. Poduska, K.M.; Regev, L.; Berna, F.; Mintz, E.; Milevski, I.; Khalaily, H.; Weiner, S.; Boaretto, E. Plaster characterization at the PPNB site of Yiftahel (Israel) including the use of ${ }^{14} \mathrm{C}$ : implications for plaster production, preservation, and dating. Radiocarbon 2012, 54, 887-896. [CrossRef]

19. Xu, B.; Hirsch, A.; Kronik, L.; Poduska, K.M. Vibrational properties of isotopically enriched materials: The case of calcite. RSC Adv. 2018, 8, 33985-33992. [CrossRef]

20. Sakai, S.; Yang, D.; Yasuda, T.; Akiyama, K.; Kuga, T.; Kano, A.; Shiraishi, F.; Amekawa, S.; Ohtsuka, S.; Nakaguchi, K.; et al. Pulsed Terahertz Radiation for Sensitive Quantification of Carbonate Minerals. ACS Omega 2019, 4, 2702-2707. [CrossRef] [PubMed]

(C) 2020 by the authors. Licensee MDPI, Basel, Switzerland. This article is an open access article distributed under the terms and conditions of the Creative Commons Attribution (CC BY) license (http://creativecommons.org/licenses/by/4.0/). 